\title{
CLINICAL AND ENDOSCOPIC PROFILE OF PEPTIC ULCER DISEASE IN LIVER CIRRHOTICS : STUDY AT A LIVER TRANSPLANT CENTRE IN SOUTH INDIA
}

\section{Gastroenterology}

Dr. MS Revathy

MD,DM Professor and Head Department of Medical Gastroenterology Government Stanley Medical College, Chennai, India

Dr. Amiya Ranjan*

MD Resident Department of Medical Gastroenterology Government Stanley Medical College, Chennai, India. * Corresponding Author

Dr. B.Sumathi

MD,DM Associate Professor Department of Medical Gastroenterology Government Stanley Medical College, Chennai, India.

Dr. Manimaran M

MD,DM Assistant Professor Department of Medical Gastroenterology Government Stanley Medical College, Chennai , India

\section{ABSTRACT}

Background: Peptic ulcer disease in patients with liver cirrhosis poses significant morbidity and mortality . It is also a cause for gastrointestinal bleed. Our study aims at providing a clinical and endoscopic profile of peptic ulcer disease in patients suffering from liver cirrhosis

Objective: Providing an insight into the clinical and endoscopic profile of patients suffering from liver cirrhosis and peptic ulcer disease, with special reference to Child Pugh score, spontaneous bacterial peritonitis, refractory ascites and other comorbidities.

Methods: This study was conducted in the Department of Medical Gastroenterology, Govt Stanley medical college, Chennai from September 2018 to April 2019. All cirrhotic patients underwent relevant blood investigations, ultrasound abdomen, portal vein doppler and upper GI endoscopy. Gastric ulcers were biopsied according to standard protocol. RUT was not performed in all cases for H.Pylori detection

Results: A total of 361 cirrhotic patients that underwent upper GI endoscopy, 45 patients were incuded in our study based on the inclusion criteria The prevalence of peptic ulcer disease in liver cirrhotics was $12.4 \%$. Males to females ratio of 38:7. Mean age was 38 years \pm 2.8 . Mean CTP score was $8.42 \pm 1.90$. median of 8 , mode 10 . Ascites was noted in 37, SBP in 22 and refractory ascites in 4 . Etiology for cirrhosis was alcohol (44.4\%), cryptogenic $(17.8 \%)$, hepatitis $\mathrm{B}(15.6 \%)$, Budd Chiari syndrome $(8.9 \%)$, hepatitis $\mathrm{C}(4.4 \%)$, wilsons disease $(2.2 \%)$, secondary biliary cirrhosis(2.2\%), autoimmune hepatitis(2.2\%). 23 had CPT score B, followed by CPT C in 15 and CPT A in 7

Conclusion: In conclusion, a significant proportion of patients with cirrhosis develop PUD. Furthermore, H pylori infection and NSAIDs are not the only, or perhaps even the most important, risk factors for PUD in patients with cirrhosis. These observations have important practical implications for physicians caring for patients with chronic liver disease

\section{KEYWORDS}

\section{INTRODUCTION}

Peptic ulcer disease (PUD) continues to have a significant impact on society, with a cumulative lifetime prevalence of $8 \%$ to $14 \%$. Peptic ulcer has been increasingly recognised in patients with cirrhosis, usually occurs without concomitant abdominal pain and often complicated by bleeding compared with the general population. Apart from delayed healing, higher rate of ulcer recurrence,other complications like perforation and gastric outlet obstruction are also more observed in these patients.

A number of studies have evaluated the possible relationships between cirrhosis and peptic ulcer disease

Our institution being a liver transplant centre under public sector in south India, catering to huge number of cirrhotic patients referred for pre liver transplant workup and its related complications like variceal bleed, refractory ascites, spontaneous bacterial peritonitis and hepato renal syndrome.

This study thus aimed at providing an insight into the endoscopic and clinical profile of patients suffering from liver cirrhosis and peptic ulcer disease, with special reference to child pugh score, spontaneous bacterial peritonitis, refractory ascites and other comorbidities

\section{METHODOLOGY}

This study was conducted in the Department of Medical Gastroenterology, Govt Stanley medical college, Chennai . This retrospective study was done based on data collected from patients undergoing endoscopy our centre from September 2019 to April 2020.

The study was approved by the Hospital's Ethics Committee.

\section{Patient Selection}

All cirrhotic patients of either sex and varied etiology undergoing screening endoscopy or for standard indications of PUD after getting informed consent were included in the study. Liver cirrhosis was diagnosed based on clinical, biochemical and imaging features. ChildPugh classification was used to assess the severity of liver disease. Ethanol as the cause was defined if there was a history of ethanol intake of over $60 \mathrm{~g} /$ day for 5 years or more and viral markers, Wilson and auto immune work up were negative.

\section{Statistical analysis}

Results are expressed as proportions for qualitative data and as mean (+-Standard Deviation) for quantitative data. SPSS software was used for all data analysis.

\section{RESULTS}

A total of 361 cirrhotic patients that underwent endoscopy, 45 patients were included in our study based on the criteria stated above. Male females ratio of 38:7. Mean age was 38 years with a standard deviation of \pm 2.8 . Mean CTP score was $8.42 \pm 1.90$. median of 8 , mode 10

Varices were classified according to standard protocol. 23 had grade II/III varices , 17 grade I varices and 5 no varices . Gastric varices were seen in 3 patients. (GOV1 1, GOV2 2). Ascites was noted in 37, SBP in 22 and refractory ascites in 4. Etiology for cirrhosis was alcohol (44.4\%), cryptogenic (17.8\%), hepatitis B(15.6\%), Budd Chiari syndrome $(8.9 \%)$, hepatitis $\mathrm{C}(4.4 \%)$, Wilsons disease $(2.2 \%)$, secondary biliary cirrhosis $(2.2 \%)$, autoimmune hepatitis(2.2\%) . 23 had CPT score B, followed by CPT C in 15 and CPT A in 7.

Location of gastric and duodenal ulcers has been graphically depicted in bar diagram 1 . Patients with duodenal bulb ulcers most commonly presented with dyspepsia, vomiting and history of upper GI bleed in the form of hematemesis or melena. Out of 19 patients with duodenal ulcers, 10 patients had ulcers in the bulb. Associated endoscopic findings in patients with duodenal bulb ulcers were antral gastritis, bulbar duodenitis, distal esophagitis, food stasis stomach, mild portal hypertensive gastropathy, partly healed ulcer and pseudodiverticulum.

Tablel shows other endoscopic findings in all cirrhotic patients Correlation of ulcers with MELD scores shows prepyloric ulcers and duodenal ulcers with higher Meld scores, compared with gastric ulcers in other locations. In this study cases were also suffering from various other comorbidities like abdominal tuberculosis, IgA nephropathy, right adnexal mass, post renal transplant graft rejection with NASH 
related cirrhosis, type 2 diabetes mellitus, growth hypopharynx, cholelithiasis and choledocholithiasis.

Table 1. Other endoscopic findings

\begin{tabular}{|l|l|}
\hline Antral gastritis & 2 \\
\hline Bulb deformed & 1 \\
\hline Bulbar duodenitis & 10 \\
\hline Congestive gastropathy & 1 \\
\hline Corpus gastritis & 1 \\
\hline Distal esophagitis & 2 \\
\hline Esophageal candidiasis grade2 & 4 \\
\hline Food stasis stomach & 3 \\
\hline Mallory Weiss tear & 2 \\
\hline Mild portal hypertensive gastropathy & 6 \\
\hline Pangastritis & 2 \\
\hline Pseudodiverticulum & 2 \\
\hline Severe portal hypertensive gastropathy & 9 \\
\hline Total & 45 \\
\hline
\end{tabular}

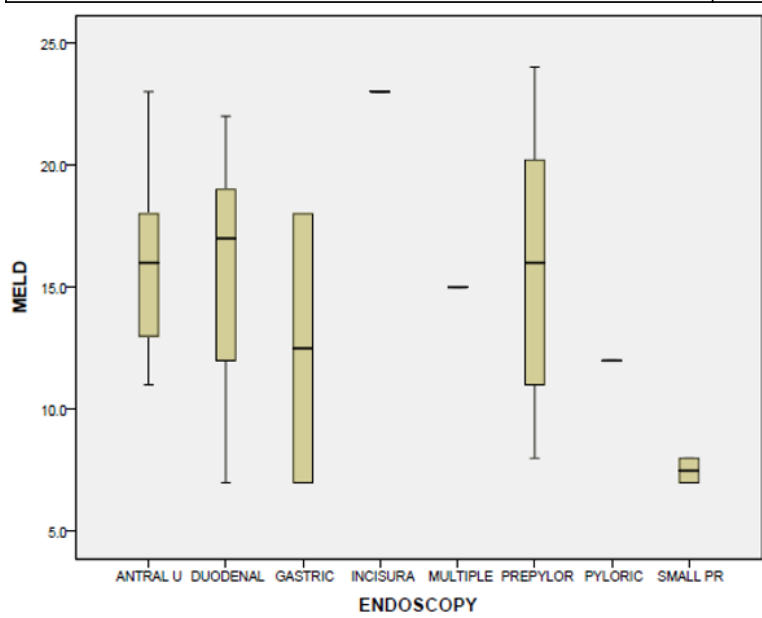

Box and Whisker plot of Distribution of ulcers and their respective MELD scores

DISTRIBUTION OF PEPTIC ULCER DISEASE IN CIRRHOTICS

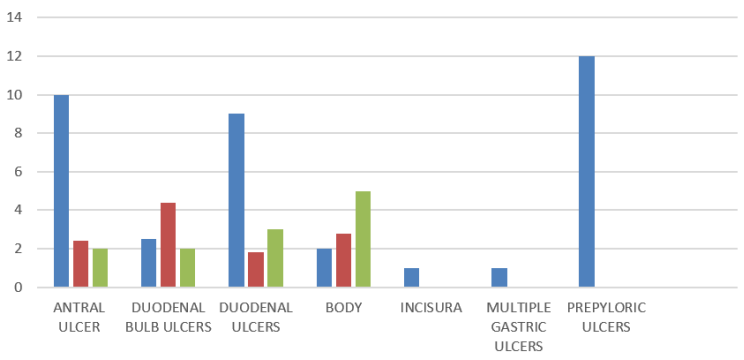

Bar Diagram showing location of gastric and duodenal ulcers

\section{DISCUSSION}

Siringo s et $\mathrm{al}^{4}$ in their study on peptic ulcer course in liver cirrhosis, peptic ulcer prevalence rates in patients with cirrhosis was $11.7 \%$, which is comparable to the results of our study . (prevalence $12.4 \%$ ). Peptic ulcer was more frequent among $\mathrm{HBsAg}+$ cirrhotics,

In another study by $\mathrm{Wu} \mathrm{CS}$ et $\mathrm{al}^{5}$, Gastric ulcers were more frequent in decompensated $(15.0 \%)$ than compensated cirrhotic patients and normal controls . In cirrhotic patients with peptic ulcer disease, Child Pugh score and ulcers was studied and most of the patients with decompensated disease presented with silent ulcers. The prevalence of peptic ulcer disease in our cirrhotic patients is also similar to this study, Child-Pugh class. Child A, B\& C 7,23 and 15 respectively .

Dore MP et $\mathrm{al}^{6}$ in their study on active peptic ulcer disease in patients with hepatitis $\mathrm{C}$ virus-related cirrhosis: the role of Helicobacter pylori infection and portal hypertensive gastropathy reported that patients with compensated cirrhosis and $\mathrm{H}$ pylori infection showed higher risk of developing a peptic ulcer. Clinical relevance of this result would be that dyspeptic patients with HCV-related cirrhosis may benefit from preventive screening and eradication of $\mathrm{H}$ pylori, especially those with features of insufficient hemostasis. Our study had this limitation that $\mathrm{H}$ pylori testing could not be done for all patients due to financial constraints .

Auroux, $\mathrm{J}$ et al ${ }^{7}$ stated that gastroduodenal ulcer was independently associated only with the severity of the gastropathy. In conclusion, in their patients with cirrhosis, the presence of gastroduodenal ulcer was significantly related to hypertensive gastropathy but not to $\mathrm{H}$. pylori infection. Recent alcohol intake favoured the occurrence of gastroduodenal erosions. In our study also alcoholic cirrhosis patient suffering from peptic ulcer disease had a high prevalence of portal hypertensive gastropathy. The grading of portal hypertensive gastropathy was done according to the $\mathrm{Mc}$

Hirchowitzin et $\mathrm{al}^{8}$ in their study also showed that peptic ulcer disease has a clinically less aggressive course in patients with liver cirrhosis as compared to the general population however the point prevalence was higher . Farinati $\mathrm{F}$ et $\mathrm{al}^{9}$ in their study tried to correlate various ulcer locations and presenting symptoms in liver cirrhotic cases and results were similar to our study, where duodenal bulbar ulcers presented with dyspepsia and antral ulcers most common symptom was a history of ugi bleed in the form of hematemesis and melena .

A review article also suggested that testing for $\mathrm{H}$ pylori in patients with cirrhosis and PUD may not be necessary ${ }^{10}$.

Though controversial, there is evidence to suggest that the incidence and the severity of PUD may be greater in those with decompensated than in those with compensated cirrhosis. The presence of ulcerrelated symptoms and the occurrence of complications seems to be related to the degree of liver function impairment. In fact, patients with asymptomatic ulcers had a more decompensated liver disease than patients with cirrhosis without ulcers or those with symptomatic ulcers.

\section{CONCLUSION}

In conclusion, a significant proportion of patients with cirrhosis develop PUD. Furthermore, H pylori infection and NSAIDs are not the only, or perhaps even the most important, risk factors for PUD in patients with cirrhosis. These observations have important practical implications for physicians caring for patients with chronic liver disease.This study also tried to bring out the relation between Meld scores and various ulcer characteristics which could give a more lucid insight into the course of progression of liver cirrhosis and the natural history of peptic ulcer disease

\section{REFERENCES}

1. Tabaqchali S, Dawson AM. Peptic ulcer and gastric secretion in patients with live disease. Gut 1964; 5: 417-21.

2. Phillips MM, Ramsby GR, Conn HO. Portacaval anastomosis and peptic ulcer: nonassociation. Gastroenterology 1975; 68: 121-31.

3. Kirk AP, Dooley JS, Hunt RH. Peptic ulceration in patients with chronic liver disease. Dig Dis Sci 1980;25: 756-60.

4. Siringo S, Burroughs AK, Bolondi L, Muia A, Di Febo G, Miglioli M, et al. Peptic ulcer and its course in cirrhosis: an endoscopic and clinical prospective study. J Hepato $1995 ; 22: 633-41$

5. Wu CS, Lin CY, Liaw YF. Helicobacter pylori in cirrhotic patients with peptic ulcer disease: a prospective, case controlled study. Gastrointest Endosc 1995;42:424-7.

6. Dore MP, Mura D, Deledda S, Maragkoudakis E, Pironti A, Realdi G. Active peptic ulce disease in patients with hepatitis $\mathrm{C}$ virus-related cirrhosis: the role of Helicobacter pylori infection and portal hypertensive gastropathy. Can J Gastroenterol 2004;18:521-4.

7. Auroux, J., Lamarque, D., Roudot-Thoraval, F. et al. Gastroduodenal Ulcer and Erosions Are Related to Portal Hypertensive Gastropathy and Recent Alcohol Intake in Cirrhotic Patients. Dig Dis Sci 48,1118-1123 (2003). https:// doi.org/

8. Hirschowitz BI, Lanas A. Atypical and aggressive upper gastrointestinal ulceration associated with aspirin abuse. J Clin Gastroenterol 2002;34: 523-8

9. Farinati F, De Bona M, Floreani A, Foschia F, Rugge M. Helicobacter pylori and the liver: any relationship? Ital J Gastroenterol Hepatol 1998; 30:124-8

10. Saad RJ, Chey WD. Peptic ulcer disease in patients with chronic liver disease: looking beyond bugs and drugs. Gastrointest Endosc. 2005;62(3):357-359. doi:10.1016/j.gie.2005.06.003 